\title{
A/D and D/A Simulation Case Development in Digital Logic Experiment Teaching
}

\author{
Yuru Zhang, Ming Zhao, Hui Li, Xiaodong Su, Haitao Jiang, Hao Jin, Yun Li, Nan Zhang \\ School of Computer and Information Engineering, Harbin University of Commerce, Harbin, China \\ hsdzyr@163.com
}

Keywords: Digital Logic; A/D; D/A; Simulated Experiment; Case Development

\begin{abstract}
Digital Logic" is a very important basic course for Software Engineering, Information Management and Information Processing, Computer Science and Technology, and Electronic Information Engineering. Because most digital logic experiment boxes do not provide $\mathrm{A} / \mathrm{D}$ and $\mathrm{D} / \mathrm{A}$ experiments which are the foundation courses for following recently, $A / D$ and D/A simulation experiment cases have been developed in this paper.
\end{abstract}

\section{Introduction}

In recent years, the data acquisition and control system formed by computer has spread to every area of daily life. Because analog signals cannot be processed by computer, it is necessary of A/D and $\mathrm{D} / \mathrm{A}$ converters to convert analog to digital and convert digital to analog in data acquisition and control systems [1].

Fig. 1 is the heating furnace temperature control system. The temperature of heating furnace will be converted into digital signal by $\mathrm{A} / \mathrm{D}$ converter and sent to the control and processing circuit. According to the deviation between the given required temperature and the actual one in the furnace, the output digital quantity would be calculated. This digital quantity is converted into analog signal by D/A converter and sent to the actuator for changing the temperature in the furnace [2].

In this case, $\mathrm{A} / \mathrm{D}$ and $\mathrm{D} / \mathrm{A}$ are the key elements which play an important role. However, the experiments of $\mathrm{A} / \mathrm{D}$ and $\mathrm{D} / \mathrm{A}$ are not usually arranged in "Digital Logic" due to the influence of class hours and equipment. In order to combine theoretical knowledge and practical application, A/D, D/A simulation experiments should be developed for other courses following.

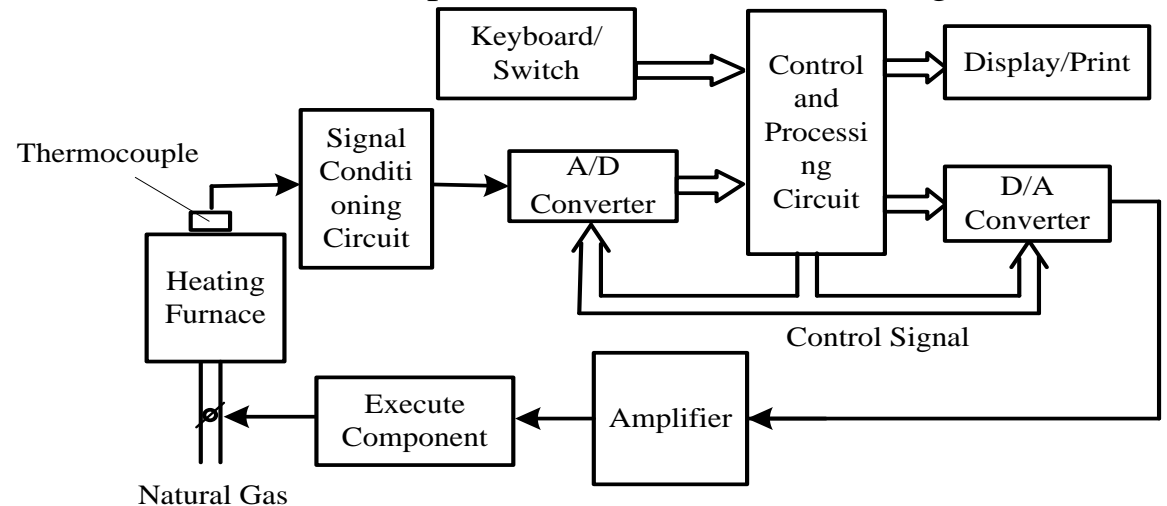

Figure.1 Heating furnace temperature control system

\section{D/A Simulation Experimental Case Development}

In this case, the 8-bit current D/A converter DAC0832 produced by NSC would be adopt. 


\subsection{Working modes of DAC0832.}

There are three working modes of DAC0832 with two latch in the chip: Cut-throught, Single buffer and Double buffer.

\subsection{Simulation case based on DAC0832}

In this case, DAC0832 is in cut-throught state. DAC0832 can realize unipolar and bipolar voltage output through external different amplifying circuits.

\subsubsection{Design of the unipolar sawtooth wave circuit.}

The output waveform of the unipolar sawtooth wave circuit is shown in Fig 2. There is a 256 system adding counter which consisted with two pieces of 74161, and the output value is 0000 0000 1111 1111 1111. The 8-bit digital quantity is converted to current signal output by D/A, then current signal will be converted to voltage signal through operational amplifier uA741. At last, the output results are displayed by oscilloscope. We can change the waveform period by CP pulse frequency. As shown in Fig.2, the output voltage signal of operational amplifier uA741 is unipolar and the voltage range is $0-5 \mathrm{~V}$.
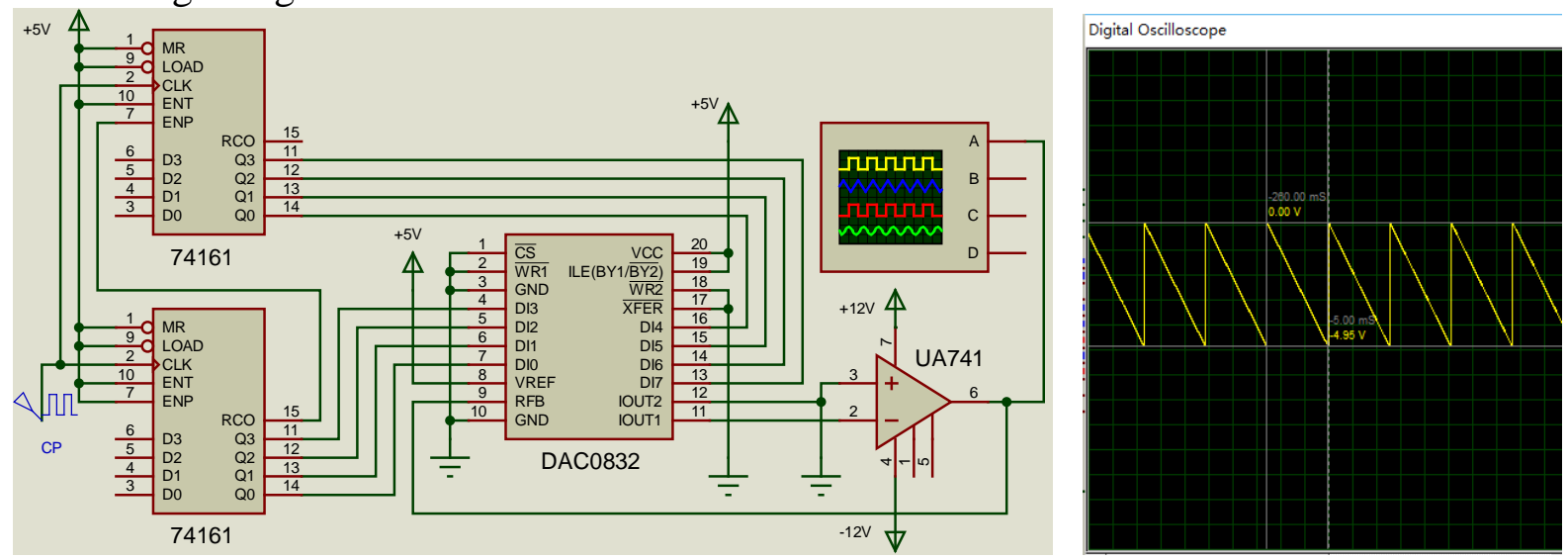

Figure.2 Output waveform of the unipolar sawtooth wave circuit

\subsubsection{Design of the bipolar sawtooth wave circuit.}

DAC0832 can realize bipolar voltage output by connecting different amplifying circuits externally based on Fig.2. The output waveform of the bipolar sawtooth wave circuit is shown in Fig 3. As shown in Fig.3, the output voltage signal is bipolar and the voltage range is $+5 \mathrm{~V} \sim-5 \mathrm{~V}$.
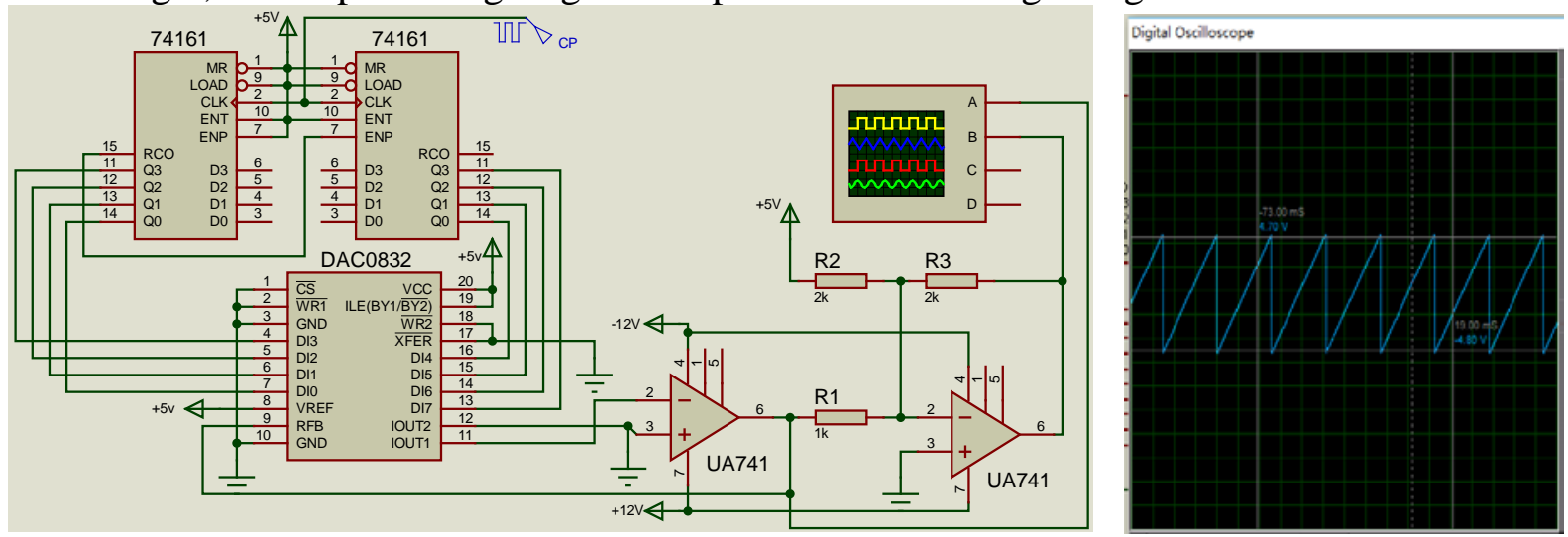

Figure.3 Output waveform of the bipolar sawtooth wave circuit

\subsubsection{Design of the unipolar sawtooth wave circuit with 555 timer.}

A clock pulse circuit composed of 555 timers [3] was designed to replace the counting pulse CLOCK in Fig.2, and the sawtooth wave period should be changed through a potentiometer used to 
adjust the clock pulse frequency. The unipolar sawtooth wave circuit with 555 timer is shown in Fig.4.

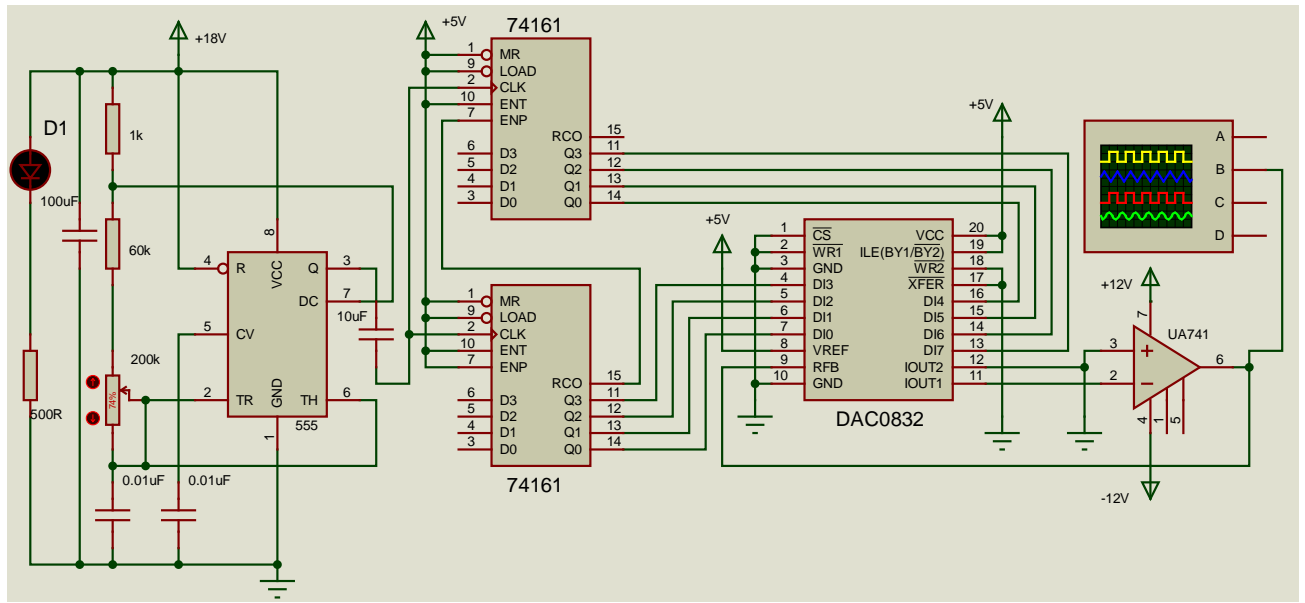

Figure.4 Unipolar sawtooth wave circuit with 555 timer

\section{A/D Simulation Experimental Case Development}

In this case, the 8-bit successive approximation ADC converter (ADC0808) produced by NSC is adopted.

\subsection{Working process of ADC0809.}

Address signal has been input, and the analog signal input channel to be converted would be determined. A/D conversion would be started, usually ALE and START are connect. Start an A/D conversion with a positive pulse.

\subsection{Simulation case based on ADC0808}

The simulation circuit based on ADC0808 is shown in Fig.5. Three address lines are connected to the ground, and IN0 channel is selected. When the analog input Vin $0=1.97 \mathrm{~V}$, the conversion result of $\mathrm{A} / \mathrm{D}$ is $01100101 \mathrm{~B}$, that is $65 \mathrm{H}$. The conversion results are shown by $8 \mathrm{LED}$ and 2 tubes. The input value should be changed with potentiometer RV1. In the circuit, the pulse CLOCK frequency is $640 \mathrm{KHz}$, and the ALE and START frequency is $1 \mathrm{~Hz}$.

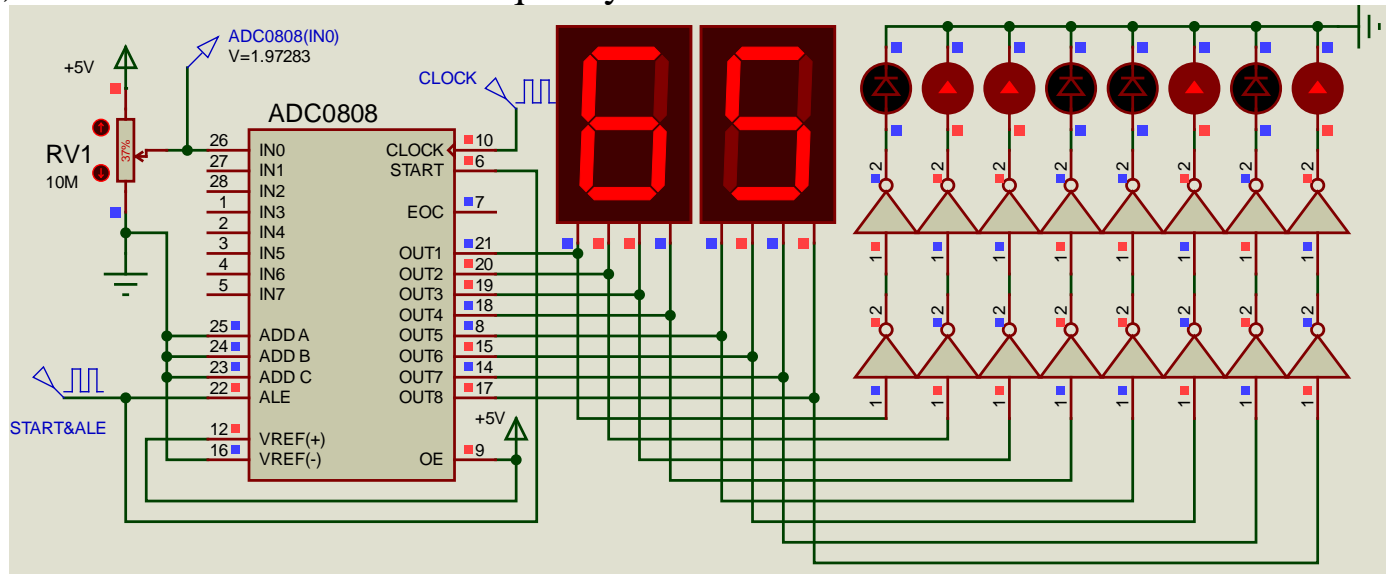

Figure.5 Simulation circuit based on ADC0808 when Vin0=1.97V

\section{Simulation Experimental Case Development Combining A/D and D/A}

The simulation circuit combining $\mathrm{A} / \mathrm{D}$ and $\mathrm{D} / \mathrm{A}$ is shown in Fig.6. There are three parts: A/D conversion circuit, D/A conversion circuit and display circuit. A/D conversion would be implemented by ADC0808, and input voltage range is $0-5 \mathrm{~V}$. The conversion results display with two 
digital tube and 8 leds display respectively. The result will be sent to DAC0832 for D/A conversion, and the conversion result will be presented the voltage probe.

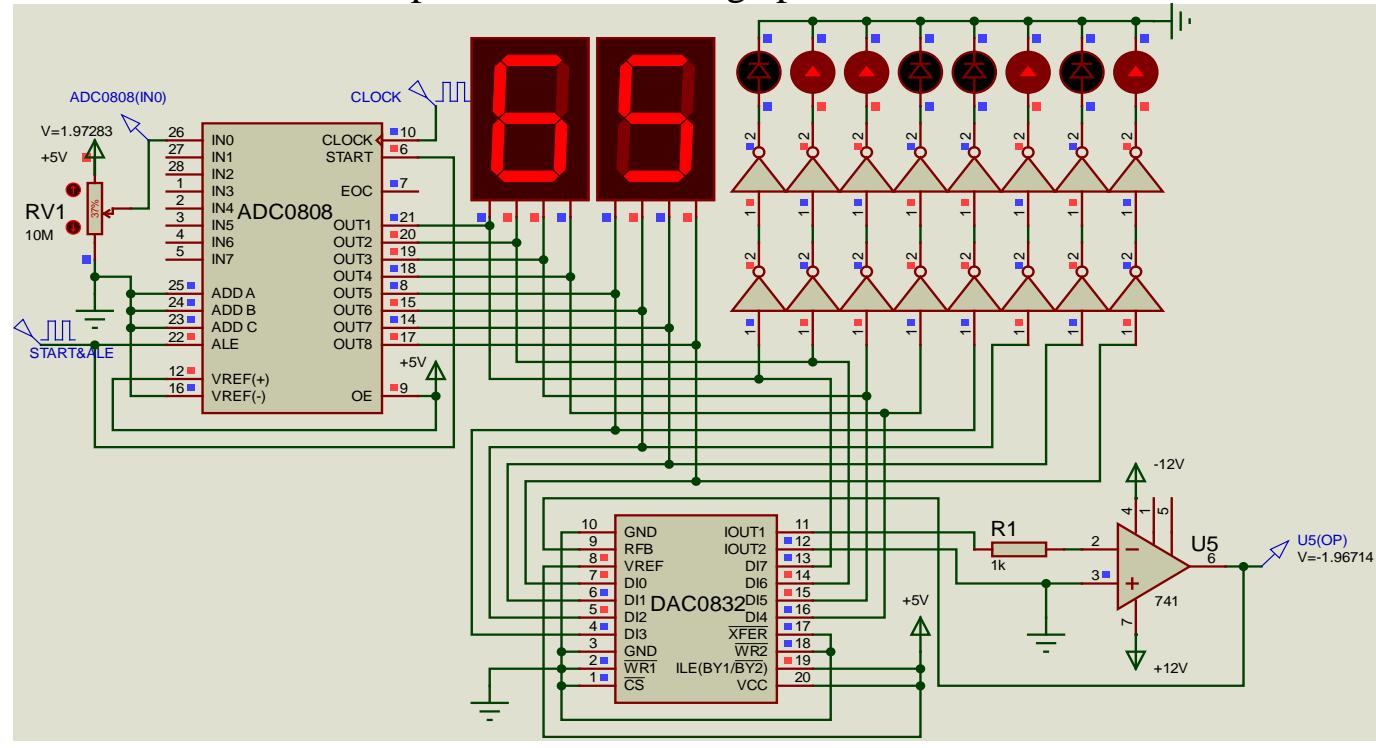

Figure.6 Simulation circuit combining A/D and D/A

As shown in Fig.6, when the analog input is $1.97283 \mathrm{~V}$, after A/D conversion and D/A conversion, the output result is $1.96714 \mathrm{~V}$ due to the conversion error.

\section{Summary}

In this paper, based on Proteus software, $\mathrm{A} / \mathrm{D}$ and $\mathrm{D} / \mathrm{A}$ simulation experiment cases have been developed. Theory knowledge and experiment content should be contented through A/D, D/A simulated experiment.

\section{Acknowledgements}

This work was financially supported by Teaching Reform Research Projects of Heilongjiang Education Department (SJGY20170055, SJGY20170075), Teaching Reform Research Projects of Harbin University of Commerce (SJXM2018A004, HSDJY014), Cooperative and Cooperative Education Project of Ministry of Education of the People's Republic of China (201601023031, 201702029029, 201702064010), Natural Science Foundation of Heilongjiang Province of China (F2015045), University Nursing Program for Young Scholars with Creative Talents in Heilongjiang Province (UNPYSCT-2016063) and Science Foundation of Harbin Commerce University (17XN060).

\section{References}

[1] Fen Dai, etal. Research on the cultivation of applied undergraduate talents in electronic information engineering [J]. China electron education. Vol1, (2016).

[2] Yuru Zhang, etal. Digital logic circuit design ( ${ }^{\text {nd }}$ edition). Harbin: Harbin Institute of Technology Press, (2018).

[3] Zhongjian Cai, Shibin Tong. Application of Proteus simulation software in the teaching of electrical courses. 4th International Conference on Education Reform and Modern Management (ERMM 2017) ISBN: 978-1-60595-478-3, (2017)

[4] Dai Fen, Wang Weixing, i Zhen, Sun Daozong, Deng Xiaoling, Jiang Sheng. Research on the Cultivation of Undergraduate Talents in Electronic and Information Engineering Specialty, China Electronics Education No.1 (2016) 
[5] Yan Gaizhen, Xu Chaoshen, Li Shuangxi. Exploration on the Engineering Education Oriented Electronic Information Specialty Construction. academic research 